\title{
NORDSTAR: paving the way for a new era in asthma research
}

\author{
Kirk Geale (1) ${ }^{1,2}$, Hatef Darabi ${ }^{1}$, Maria Lindh ${ }^{1}$, Hanna Fues Wahl ${ }^{1}$, \\ Oskar Ström ${ }^{1,3}$, Hui Cao ${ }^{4}$, Luisa Alvares ${ }^{5}$, Rikke Dodge ${ }^{6}$, Emil Loefroth ${ }^{7}$, \\ Alan Altraja $\mathbb{1}^{8}$, Vibeke Backer ${ }^{9}$, Helena Backman (10 ${ }^{10}$, Leif Bjermer ${ }^{11}$, \\ Apostolos Bossios ${ }^{12,13}$, Bernt Bøgvald Aarli ${ }^{14}$, Barbro Dahlén ${ }^{15}$, Ole Hilberg ${ }^{16}$, \\ Christer Janson (10 ${ }^{17}$, Hannu Kankaanranta (10 ${ }^{18}$, Jussi Karjalainen ${ }^{19}$, \\ Paula Kauppi ${ }^{20}$, Maritta Kilpeläinen ${ }^{21,22}$, Sverre Lehmann ${ }^{23}$, \\ Lauri Lehtimäki ${ }^{19,24}$, Bo Lundbäck ${ }^{25}$, Thomas Sandström ${ }^{10}$, \\ Charlotte Suppli Utrik ${ }^{26}$, Asger Sverrild ${ }^{27}$, Arja Viinanen (1021,22, \\ Anna von Bülow ${ }^{28}$, Valentyna Yasinska ${ }^{29}$ and Celeste Porsbjerg (1) ${ }^{30}$
}

Affiliations: ${ }^{1}$ Quantify Research, Stockholm, Sweden. ${ }^{2}$ Dept of Public Health and Clinical Medicine, Umeå University, Umeå, Sweden. ${ }^{3}$ Dept of Learning, Informatics, Management and Ethics (LIME), Medical Management, Stockholm, Sweden. ${ }^{4}$ Novartis Pharmaceuticals Corporation, East Hanover, NJ, USA. ${ }^{5}$ Novartis Pharma AG, Basel, Switzerland. ${ }^{6}$ Novartis Pharmaceuticals A/S, Copenhagen, Denmark. ${ }^{7}$ Novartis Sverige AB, Kista, Sweden. ${ }^{8}$ Dept of Pulmonary Medicine, University of Tartu, and Dept of Pulmonary Medicine, Tartu University Hospital Tartu, Estonia. ${ }^{9}$ Centre for Physical Activity Research, Rigshospitalet and Copenhagen University, Copenhagen, Denmark. ${ }^{10}$ Dept of Public Health and Clinical Medicine, University Hospital of Umeå, Umeå University, Umeå, Sweden. ${ }^{11}$ Respiratory Medicine and Allergology, Dept of Clinical Sciences, Lund, Sweden. ${ }^{12}$ Dept of Respiratory Medicine and Allergy, Karolinska University Hospital Huddinge, Stockholm, Sweden. ${ }^{13}$ Dept of Medicine, Huddinge, Karolinska Institutet, Stockholm, Sweden. ${ }^{14}$ Dept of Clinical Science, University of Bergen, Haukeland University hospital, Bergen, Norway. ${ }^{15}$ Dept of Medicine, Karolinska University Hospital Huddinge and Karolinska Institutet, Stockholm, Sweden. ${ }^{16}$ IRS-center, Lillebælt Hospital, Medicine, Vejle, Denmark. ${ }^{17}$ Dept of Medical Sciences, Respiratory, Allergy and Sleep Research, Uppsala University, Uppsala, Sweden. ${ }^{18}$ Seinäjoki Central Hospital, Seinäjoki, Finland. ${ }^{19}$ Allergy Centre, Tampere University Hospital, Tempere, Finland. ${ }^{20}$ Dept of Allergy, Respiratory Diseases and Allergology, University of Helsinki and Helsinki University Hospital, Helsinki, Finland. ${ }^{21}$ Division of Medicine, Dept of Pulmonary Diseases, Turku University Hospital, Turku, Finland. ${ }^{22}$ Dept of Pulmonary Diseases and Clinical Allergology, University of Turku, Turku, Finland. ${ }^{23}$ Haukeland University Hospital, Bergen, Norway. ${ }^{24}$ Faculty of Medicine and Health Technology. Tampere University, Tampere, Finland. ${ }^{25}$ Dept of Internal Medicine at Institute of Medicine/Krefting Research Centre, University of Gothenburg and Sahlgrenska University Hospital, Gothenburg. Sweden. ${ }^{26}$ Dept of Respiratory Medicine, Hvidovre University Hospital and Institute of Clinical Medicine, University of Copenhagen, Copenhagen, Denmark. ${ }^{27}$ Dept of Respiratory Medicine, Bispebjerg Hospital, Copenhagen University, Copenhagen, Denmark. ${ }^{28}$ Dept of Respiratory Medicine, Bispebjerg and Frederiksberg Hospital, Copenhagen, Denmark. ${ }^{29}$ Dept of Respiratory Medicine and Allergy, Karolinska University Hospital, Stockholm, Sweden. ${ }^{30}$ Dept of Respiratory Medicine, Respiratory Research Unit, Bispebjerg Hospital, Copenhagen, Denmark.

Correspondence: Kirk Geale, Umeå Universitet Medicinska fakulteten, Public Health and Clinical Medicine, Umeå University, Umeå, 901 87, Sweden. E-mail: kirk.gealedumu.SE

@ERSpublications

NORDSTAR is a longitudinal dataset for the study of asthma comprising 3.3 million individuals and over 50 million person-years in 4 Nordic countries. The data include diagnoses, medication dispensing, use of resources and costs, socio-demographics, and mortality. http://bit.ly/2HueyEt

Cite this article as: Geale $\mathrm{K}$, Darabi $\mathrm{H}$, Lindh $\mathrm{M}$, et al. NORDSTAR: paving the way for a new era in asthma research. Eur Respir J 2020; 55: 1902476 [https://doi.org/10.1183/13993003.02476-2019]. 


\section{Introduction}

Asthma is a heterogeneous, chronic airway disease with large variation in prevalence between geographies $[1,2]$. In the Nordic countries, prevalence of asthma has been reported to be $10 \%$ in Denmark [1], $9-11 \%$ in Finland [1, 3], 11-12\% in Norway [1], and $8-10 \%$ in Sweden [1, 4, 5]. Poor asthma control is associated with substantial reductions in patient quality of life [6-8], and higher morbidity and mortality [9, 10], together with significantly increased direct and indirect costs $[6,11]$.

Comprehensive longitudinal follow-up of asthma patients in routine clinical care is essential to understand the nature of the disease, patient characteristics, and long-term trajectories to ultimately improve patient outcomes. Although many valuable asthma research datasets exist today [12-17], they typically include a limited number of follow-up visits involving clinical assessments for a modest number of patients, and only the Danish National Database for Asthma [18] comprises a nationwide population. In contrast, real-world assessments of entire asthma populations enable descriptions and assessments of patients, disease and provision of healthcare in routine clinical care.

Each Nordic country maintains nationwide administrative and medical registries that are uniquely suited to studying patients at a population level. For decades, the Nordic countries have enforced mandatory reporting into each administrative registry, enabling complete, longitudinal coverage of each country's population in a real-world context [19] that is not possible in many other countries [20]. The automatic and standardised registration of all residents avoids the burden associated with study participant enrolment and administration [20], effectively minimising resource use and cost. A unique personal identification number (PIN) given to all Nordic residents can be used to link each dataset together at the patient level which aids in avoiding selection bias, which often limits epidemiological studies. Similarities in content and in reporting processes into these Nordic registries facilitates the use of comparable asthma-related definitions, such as severity and control, in addition to consistency in interpretation of results between countries [21].

To the best of our knowledge, there is no existing data containing long-term longitudinal follow-up of asthma populations in routine clinical care across multiple countries built on nationwide data. A multinational collaboration was initiated using the NORdic Dataset for aSThmA Research (NORDSTAR) to pursue a common goal of improving asthma patients' quality of life, by bringing together representatives active in clinical practice, academic research, and the pharmaceutical industry. To date, NORDSTAR includes patient-level information from four Nordic countries (Denmark, Finland, Norway, and Sweden). The purpose of this work is to describe NORDSTAR in terms of the patients included, research goals, data sources and contents, and administration of the data as a basis for future research and collaboration.

\section{NORDSTAR data}

The NORDSTAR data was retrieved across several years involving a variety of ethical boards, data holders, and research institutions. Ethical approvals were obtained according to the local and EU law and positive decisions regarding personal data protection assessments were received from the responsible authorities in each country. Informed consent from individual patients is not required for registry studies on retrospective data in the Nordics and was therefore not obtained.

Each country included in NORDSTAR maintains administrative, population-based registries with complete nationwide coverage. Healthcare is publicly financed and contacts with the public healthcare system must be reported by law $[20,21]$. These data collection systems are independently and continuously maintained with longitudinal, individual-level information.

In each of the respective countries, patient-level linkage between each administrative registry was conducted. The national organisations responsible for creating these statistics were: Statistics Denmark, the Swedish National Board of Health and Welfare, and Statistics Finland. No patient-level linkage between registries was performed for data from Norway as all data was extracted from a single registry, the Norwegian Prescribed Drug Registry. The unique PIN provided to each resident is assigned at birth or immigration and subsequently used throughout the patient's life. All data reported into the national registries include the PIN, enabling the extraction and amalgamation of patient-level data across all relevant data sources within each country. All data was pseudonymised before delivery to the research group.

A description of the NORDSTAR variables is presented in table 1.

\section{Patient population}

Individuals were included in NORDSTAR if they fulfilled at least one of the two following criteria between 2006 and the latest available year of data: 


\begin{tabular}{|c|c|c|c|c|}
\hline Variable type & Denmark ${ }^{\#}$ & Finland" & Norway $^{+}$ & Sweden $\S$ \\
\hline \multicolumn{5}{|l|}{ Demographics } \\
\hline Year of birth & $x$ & $x$ & $x$ & $x$ \\
\hline Sex & $x$ & $x$ & $x$ & $x$ \\
\hline \multicolumn{5}{|l|}{ Geography } \\
\hline Availability & $1986-2016$ & $1992-2016$ & $2004-2018$ & $1987-2017$ \\
\hline Migration date and type & $x$ & & & $x$ \\
\hline Municipality of residence & $x$ & & & $x$ \\
\hline County of residence & $x$ & $x$ & $x$ & $x$ \\
\hline \multicolumn{5}{|l|}{ Death } \\
\hline Availability & $2004-2014$ & $2006-2015$ & $2007-2018$ & 2006-2017 \\
\hline Date of death & $x$ & $x$ & $x$ & $x$ \\
\hline Cause of death & $x$ & $x$ & & $x$ \\
\hline \multicolumn{5}{|l|}{ Socioeconomics } \\
\hline Availability & $1990-2015$ & $1987-2015$ & NA & 2004-2014 \\
\hline Individual disposable income ${ }^{f}$ & $x$ & $x$ & & $x$ \\
\hline Family disposable income $e^{f}$ & $x$ & $x$ & & $x$ \\
\hline Sickness benefit $f$ & & $x$ & & $x$ \\
\hline Welfare benefit ${ }^{f}$ & & $x$ & & $x$ \\
\hline Employment status & $x$ & $x$ & & $x$ \\
\hline Number of days unemployed & & & & $x$ \\
\hline Number of days with benefits & & & & $x$ \\
\hline Education level & $X$ & $x$ & & $\mathrm{X}$ \\
\hline \multicolumn{5}{|l|}{ Primary healthcare } \\
\hline Availability & NA & $2011-2016$ & NA & NA \\
\hline Date registered for care contact & & $x$ & & \\
\hline Primary care diagnoses (ICD-10, ICPC2) & & $x$ & & \\
\hline Procedure codes & & $x$ & & \\
\hline \multicolumn{5}{|l|}{ Secondary healthcare } \\
\hline Availability & $1995-2014$ & $1992-2015$ & NA & $1987-2015$ \\
\hline Date registered for care contact/hospitalisation & $x$ & $x$ & & $x$ \\
\hline Date discharged & $x$ & $x$ & & $x$ \\
\hline Secondary care diagnoses & $x$ & $x$ & & $X$ \\
\hline Type of clinic discharged from (e.g. emergency) & & $x$ & & $x$ \\
\hline Procedure codes & $x$ & $x$ & & $x$ \\
\hline Planned or unplanned visit indicator & $x$ & & & $x$ \\
\hline Inpatient or outpatient indicator & $x$ & $x$ & & $x$ \\
\hline $\begin{array}{l}\text { Date and procedure code for hospital-administered } \\
\text { monoclonal antibody } \# \#\end{array}$ & $x$ & & & \\
\hline \multicolumn{5}{|l|}{ Pharmacy-dispensed medications ${ }^{\text {กा }}$} \\
\hline Availability & $1995-2015$ & $2011-2016$ & $2004-2018$ & $2005-2017$ \\
\hline ATC code, product name and substance & $x$ & $x$ & $x$ & $x$ \\
\hline Date of dispensed prescription at pharmacy & $x$ & $x$ & $x$ & $x$ \\
\hline Prescribing physician speciality & $x$ & & & $x$ \\
\hline Number of dispensed packages & $x$ & $x$ & $x$ & $x$ \\
\hline Inhaler type for asthma drugs & $x$ & $x$ & $x$ & $x$ \\
\hline Package size and package daily defined doses & $x$ & $x$ & $x$ & $x$ \\
\hline $\begin{array}{l}\text { Amount of dispensed medication (e.g. number of } \\
\text { milligrams, millilitres, etc.) }\end{array}$ & $x$ & $x$ & $x$ & $x$ \\
\hline $\begin{array}{l}\text { Diagnosis code associated with prescription } \\
\text { (primary and secondary) }\end{array}$ & & & $x$ & \\
\hline
\end{tabular}

ATC: anatomical therapeutic chemical, ICD: international classification of disease, ICPC: international classification of primary care, NA: not applicable. ": Danish Health Authority, Statistics Denmark; ๆ: National Institute for Health and Welfare, Social Insurance Institution of Finland; ${ }^{+}$: Norwegian Institute of Public Health; ${ }^{\S}$ : The National Board of Health and Welfare, Statistics Sweden; ${ }^{f}$ : in local currency; \#\#: no information on the amount of drug administered nor other dosing information is available for hospital-administered monoclonal antibodies, only ATC or monoclonal antibody-specific procedure code and date of administration; ๆा: only available for pharmacy-dispensed medications, not for hospital-administered medications. 
1) Inclusion criterion 1 (diagnosis): One or more asthma diagnosis (international classification of disease (ICD)-10: J45/J46) registered in secondary care. (In Norway, ICD-10 diagnosis codes registered in association with prescriptions were used, as no data is available for patients visiting secondary care centres.)

2) Inclusion criterion 2 (medication): Dispensation with respiratory drugs (anatomical therapeutic chemical (ATC): R03.xx.xx) on $\geqslant 2$ occasions separated by at most 12 months.

A registered diagnosis of asthma in secondary care (inclusion criterion 1) is a reliable indicator of asthma, and similar medication-based proxy criteria for asthma (inclusion criterion 2) have previously been used $[9,22]$ and validated [23]. It is important to note that the diagnostic inclusion criterion applies only to patients treated in secondary care while the medication proxy criteria applies to patients receiving prescriptions originating from both primary and secondary care.

Despite the support from the literature for the inclusion criteria above, there are patient groups that may be misclassified and therefore the authors recommend the application of exclusion criteria to reduce the likelihood of including non-asthma patients in the population. In doing so, researchers must trade some degree of sensitivity for specificity with the balance varying by research project. Below are the key groups of patients at risk of inclusion, who may not be true asthma patients, alongside the authors' recommendation for how to exclude those patients using definitions applicable to NORDSTAR:

1) Treatment for lower chronic respiratory conditions other than asthma managed in secondary care: patients dispensing respiratory medication with no asthma diagnosis, but with a diagnosis of another chronic lower respiratory disease.

2) Possible COPD treated in primary care: Patients aged $\geqslant 45$ years dispensing COPD medication (fixed combination long-acting muscarinic-antagonists/long-acting $\beta$-antagonists (LABA), tiotropium Spiriva HandiHaler (not Spiriva Respimat), umeclidinium, aclidinium, glycopyrronium) with no chronic lower respiratory disease diagnosis (including asthma).

3) Suspected urticaria: Patients with a diagnosis of urticaria in secondary care, but no chronic lower respiratory disease diagnoses, in addition to treatment with omalizumab.

It is important to note that the suggested exclusion criteria do not aim to exclude asthma patients with comorbid conditions; the purpose is to exclude those who appear to be asthma patients according to the NORDSTAR inclusion criteria, but due to the features listed above are likely to primarily suffer from other non-asthma indications that use respiratory medicines.

The full NORDSTAR population includes a total of 3301773 patients where 799347 were identified in Denmark (24\%), 232713 in Finland (7\%), 853450 in Norway (26\%) and 1416263 in Sweden (43\%). Female patients comprised 52\%,55\%,52\%, and 54\% of each country's population, respectively. The inclusion period differs according to the initiation of relevant registries in each country, but the overall NORDSTAR identification period occurred between 2004 and 2018. In all countries, over 65\% of all patients included in NORDSTAR could be identified according to the medication inclusion criterion alone (two dispensations with ATC code: R03+ within 12 months of each other).

\section{Collaborative platform}

NORDSTAR is supported by a multi-party, collaborative research platform comprising clinical expertise, pharmaceutical manufacturer perspectives and data analytics competence: The Nordic Severe Asthma Network (NSAN) is a network of severe asthma specialists from the Nordic countries focusing on improving the diagnostics and treatment of severe and possible severe asthma. In the NORDSTAR project, the members of NSAN provide clinical insight to the interpretation of register data. The pharmaceutical industry partners provide insights into research and development and patient access. Quantify Research maintains and secures data, performs data management and analysis, and expedites medical communications. The groups working with NORDSTAR welcome future collaboration with other stakeholders.

\section{Remarks and context}

NORDSTAR is a unique, multinational initiative in the Nordics enabling longitudinal follow-up of asthma patients in contemporary, routine clinical care. This population-based data provides a basis for comprehensive characterisation of all Nordic asthma patients and its longitudinal nature enables the study of temporal trends and identification of asthma-related events over patients' life-course. The data is well suited to research aiming to characterise and categorise asthma patients, study comparative effectiveness, analyse health economics and healthcare burden, describe patient and treatment pathways, and to provide a basis for comparisons between Nordic countries. NORDSTAR is unique in that it includes many patients 
from early stages of the disease through to potential severe asthma. The multinational aspect of the data also enables the study of certain structural differences between healthcare systems.

There are a variety of complexities and subtleties related to the secondary healthcare data included in NORDSTAR, which include patient composition, provision of treatment and care, patient registration, and data collection procedures, as well as interpretation of variables and outcomes between countries. Each of these is nuanced and differ over time and geography. It is therefore essential that a collaborative, multidisciplinary team is involved in the analysis of NORDSTAR data. The asthma healthcare professionals working alongside NORDSTAR, the NSAN, ensure that clinical perspectives and relevance are at the forefront of prioritising, designing, and interpreting NORDSTAR-based research.

Diagnostic, phenotypic, and prognostic markers associated with the asthma disease course include forced expiratory volume in $1 \mathrm{~s}$, tobacco usage, atopy and airway inflammation. These risk factors are not currently available in NORDSTAR. This limitation implies that classifying patients into asthma severity and control categories must be done using proxy definitions tailored to administrative registry data instead of exact implementations using European Respiratory Society/American Thoracic Society [24] or Global Initiative for Asthma [25] guidelines. This requires careful design and implementation of proxy measures, ideally those which have been validated. However, severe asthma definitions based on medication use, i.e. high use of inhaled corticosteroids and leukotriene receptor antagonists and/or LABA, have been used efficiently in recent large epidemiological studies [9, 26-28].

NORDSTAR data exists alongside a variety of high-quality clinical cohorts in the Nordic countries containing many of the non-administrative variables discussed above, likely including many of the same patients within the same time frames as NORDSTAR. In this respect, NORDSTAR acts as a compliment to existing clinical data. In the future, some of these clinical cohorts may be added to NORDSTAR to extend the research possibilities. Study of additional research questions may require new ethical approvals and/or data extractions. At the time of writing, the existing NORDSTAR data was being updated to ensure it remains contemporary as treatment and patient landscapes change.

While many aspects of Nordic healthcare systems and administrative data are similar, Finland began a progressive transition to mandatory prescriber-registered electronic prescriptions during patient follow-up. Consequently, the coverage of patient prescriptions in NORDSTAR changes significantly over time for Finnish patients, reaching full coverage in approximately 2017. This is important for two reasons. First, since more patients are identified and included through the medication criterion than the diagnosis criterion, there is less opportunity, for administrative reasons, for Finnish patients to be identified overall resulting in relatively low patient numbers when compared with Denmark, Norway and Sweden, even after adjusting for each country's population size. Second, patients treated in primary care were identified in NORDSTAR via the prescription-based inclusion criteria only. Since this data is under-represented in Finland, it is expected that Finnish NORDSTAR patients are biased towards being more severe. The authors recommend the use of qualified statements, when making inter-country comparisons in general, and especially regarding Finland.

Currently, the Norwegian NORDSTAR data is limited to the prescription registry. However, Norway is unique in that the diagnosis codes in primary and secondary care are registered alongside prescriptions. Norway is the only country in NORDSTAR where the prescriptions can be directly linked to ICD diagnoses codes. While this is a useful attribute in many settings, reimbursement regulations may impact the way in which diagnoses are set for patients when reimbursement is linked to an asthma or COPD diagnosis, but not both. Norwegian asthma patients who did not fill a prescription for respiratory medication are not included in the NORDSTAR population, nor can patients' full comorbidity profile be assessed, since the diagnosis data is only available when linked to a prescription. Despite these shortcomings, it is expected that there is little selection bias in Norway, since few asthma patients receive only secondary care diagnoses, but no prescribed medication. Socioeconomic data is not available in Norway.

Monoclonal antibodies (MABs) indicated for severe asthma (benralizumab, dupilumab, mepolizumab, omalizumab and reslizumab at the time of writing) are usually administered in the hospital setting as opposed to secondary care clinics in the Nordic countries. Hospital-administered drugs are not directly dispensed to patients and are therefore not fully registered in the Finnish, Norwegian or Swedish data. The data on hospital-administered medication is available in Denmark via ATC and procedures codes in the Danish patient registry but lacks information on dosing and amount of drug administered. In future updates of the NORDSTAR data, the coverage of MAB medication is expected to improve, as the hospital-based administration of MABs becomes less prevalent in favour of pharmacy-dispensed pre-filled syringes for home use. 
The data included in NORDSTAR are clearly relevant to the Nordic context, but many aspects of NORDSTAR-based research are generalisable to other settings. Epidemiological study of patient characteristics, patient trajectories, and treatment effectiveness are likely to be highly applicable to other Western patient groups. Research related to provision of healthcare including treatment patterns, costs, and resource use may be combined with expert advisory boards, such as Delphi panels to promote cross-national applications. This approach may be best suited to non-Nordic candidate populations that also operate within single-payer healthcare systems. Finally, NORDSTAR is an inspiring example of a successful collaboration in the era of big data between different stakeholders including academia, the pharmaceutical industry, and government authorities, that could drive large amounts of innovative research for the benefit of patients.

NORDSTAR is a population-based multinational Nordic dataset developed alongside active research collaboration with Nordic asthma specialists through the NSAN, for conducting contemporary, real-world research on asthma patients to improve clinical practice and patients' quality of life.

Acknowledgements: The authors thank Thomas Fast at the Institute of Applied Economics and Health Research for collaboration in the extraction and analysis of NORDSTAR data in Denmark.

Conflict of interest: K. Geale reports research project funding from Novartis for the current work; personal fees for consultancy from Quantify Research, during the conduct of the study and outside the submitted work. H. Darabi reports research project funding from Novartis for the current work; personal fees for consultancy from Quantify Research, during the conduct of the study and outside the submitted work. M. Lindh reports research project funding from Novartis for the current work; personal fees for consultancy from Quantify Research, during the conduct of the study and outside the submitted work. H. Fues Wahl reports research project funding from Novartis for the current work; personal fees for consultancy from Quantify Research, during the conduct of the study and outside the submitted work. O. Ström reports research project funding from Novartis for the current work; personal fees for consultancy from Quantify Research, during the conduct of the study and outside the submitted work. H. Cao is an employee of Novartis Pharmaceutical Company. L.A. Alvares is an employee of Novartis. R. Dodge has nothing to disclose. E. Loefroth is an employee of Novartis. A. Altraja reports personal fees and travel and educational support from AstraZeneca, Berlin-Chemie Menarini, Boehringer Ingelheim, CSL Behring, Roche, Teva and Sanofi, grants, personal fees and travel and educational support from Chiesi (Norameda) and GlaxoSmithKline, personal fees and travel support from MSD, grants and personal fees from Bayer, travel and educational support from Actelion (Johnson and Johnson), personal fees from Shire Pharmaceuticals, outside the submitted work. V. Backer has nothing to disclose. H. Backman reports personal fees for lectures from AstraZeneca, outside the submitted work. L. Bjermer reports personal fees from AstraZeneca, Boehringer, Chiesi, GSK, Novartis, Teva and ALK, outside the submitted work. A. Bossios reports personal fees for advisory board work and/or lectures from AZ, Teva, GSK and Novartis, outside the submitted work. B. Bøgvald Aarli reports personal fees for lectures from Boehringer Ingelheim, Novartis, AstraZeneca, Chiesi and Alere, personal fees for consultancy from GlaxoSmithKline, and has stock in from KBB Medic AS, outside the submitted work. B. Dahlén reports personal fees for lectures and advisory board work from AstraZeneca, GSK, Teva, Sanofi and Novartis, outside the submitted work. O. Hilberg has nothing to disclose. C. Jansson has nothing to disclose. H. Kankaanranta reports grants, personal fees for lectures and consultancy, and non-financial support to attend meetings from AstraZeneca, personal fees for lectures and consultancy from Chiesi Pharma AB, Novartis and GlaxoSmithKline, personal fees for lectures and consultancy, and non-financial support to attend meetings from Boehringer Ingelheim and Orion Pharma, personal fees for lectures from Mundipharma, personal fees for consultancy from Sanofi Genzyme, outside the submitted work. J. Karjalainen reports personal fees from AstraZeneca, Boehringer Ingelheim, Chiesi, GlaxoSmithKline, Mundipharma, Novartis, Orion Pharma and Teva, outside the submitted work. P. Kauppi reports personal fees for lectures from GSK, Teva and Novartis, personal fees for manuscript preparation from Fimea, personal fees for consultancy from Sanofi, outside the submitted work. M. Kilpeläinen has nothing to disclose. S. Lehmann reports data analysis and manuscript preparation support from Novartis, during the conduct of the study; personal fees for lectures from AstraZeneca, personal fees for manuscript preparation from Boehringer Ingelheim, outside the submitted work. L. Lehtimäki reports personal fees from AstraZeneca, Chiesi, GSK, Boehringer Ingelheim, MSD, Novartis, pi tlsb -.005wOrionPharma, ALK, Teva and Sanofi, outside the submitted work. B. Lundbäck reports grants from AstraZeneca, personal fees for lectures and advisory board work from AstraZeneca, Novartis, GSK and Sanofi, outside the submitted work. T. Sandström has nothing to disclose. C. Suppli. Ulrik has nothing to disclose. A. Sverrild has nothing to disclose. A. Viinanen has nothing to disclose. A. von Bülow has nothing to disclose. V. Yasinska has nothing to disclose. C. Porsbjerg reports grants and personal fees from AstraZeneca, GSK, Novartis, Sanofi and Teva, outside the submitted work.

Support statement: This work was funded by Novartis Pharma. Funding information for this article has been deposited with the Crossref Funder Registry.

\section{References}

1 To T, Stanojevic S, Moores G, et al. Global asthma prevalence in adults: findings from the cross-sectional world health survey. BMC Public Health 2012; 12: 204.

2 Braman SS. The global burden of asthma. Chest 2006; 130: 1 Suppl., 4s-12s.

3 Honkamaki J, Hisinger-Molkanen H, Ilmarinen P, et al. Age- and gender-specific incidence of new asthma diagnosis from childhood to late adulthood. Respir Med 2019; 154: 56-62.

4 Backman H, Räisänen P, Hedman L, et al. Increased prevalence of allergic asthma from 1996 to 2006 and further to 2016-results from three population surveys. Clin Exp Allergy 2017; 47: 1426-1435.

5 Bjerg A, Ekerljung L, Middelveld R, et al. Increased prevalence of symptoms of rhinitis but not of asthma between 1990 and 2008 in Swedish adults: comparisons of the ECRHS and GA(2)LEN surveys. PloS One 2011; 6: e16082. 
6 Bahadori K, Doyle-Waters MM, Marra C, et al. Economic burden of asthma: a systematic review. BMC Pulm Med 2009; 9: 24.

7 Chen S, Golam S, Myers J, et al. Systematic literature review of the clinical, humanistic, and economic burden associated with asthma uncontrolled by GINA Steps 4 or 5 treatment. Curr Med Res Opin 2018; 34: 2075-2088.

8 Jansson S-A, Axelsson M, Hedman L, et al. Subjects with well-controlled asthma have similar health-related quality of life as subjects without asthma. Respir Med 2016; 120: 64-69.

9 von Bulow A, Kriegbaum M, Backer V, et al. The prevalence of severe asthma and low asthma control among Danish adults. J Allergy Clin Immunol Pract 2014; 2: 759-767.

10 Suissa S, Ernst P. Inhaled corticosteroids: impact on asthma morbidity and mortality. J Allergy Clin Immunol 2001; 107: 937-944.

11 Janson C, Lisspers K, Stallberg B, et al. Health care resource utilization and cost for asthma patients regularly treated with oral corticosteroids - a Swedish observational cohort study (PACEHR). Respir Res 2018; 19: 168.

12 Fleming L, Murray C, Bansal AT, et al. The burden of severe asthma in childhood and adolescence: results from the paediatric U-BIOPRED cohorts. Eur Respir J 2015; 46: 1322-1333.

13 Hyrkas H, Ikaheimo TM, Jaakkola JJ, et al. Asthma control and cold weather-related respiratory symptoms. Respir Med 2016; 113: 1-7.

14 Kupczyk M, Haque S, Sterk PJ, et al. Detection of exacerbations in asthma based on electronic diary data: results from the 1-year prospective BIOAIR study. Thorax 2013; 68: 611-618.

15 Lötvall J, Ekerljung L, Rönmark EP, et al. West Sweden Asthma Study: Prevalence trends over the last 18 years argues no recent increase in asthma. Respir Res 2009; 10: 94.

16 Teague WG, Phillips BR, Fahy JV, et al. Baseline features of the Severe Asthma Research Program (SARP III) cohort: differences with age. J Allergy Clin Immunol Pract 2018; 6: 545-554.

17 Tuomisto E L, Kankaanranta H, Ilmarinen P, et al. Seinäjoki Adult Asthma Study (SAAS): a protocol for a 12 -year real-life follow-up study of new-onset asthma diagnosed at adult age and treated in primary and specialised care. NPJ Prim Care Respir Med 2015; 25: 1-4.

18 Backer V, Lykkegaard J, Bodtger U, et al. The Danish National Database for Asthma. Clin Epidemiol 2016; 8: 601-606.

19 Gavrielov-Yusim N, Friger M. Use of administrative medical databases in population-based research. J Epidemiol Community Health 2014; 68: 283-287.

20 Ludvigsson JF, Haberg SE, Knudsen GP, et al. Ethical aspects of registry-based research in the Nordic countries. Clin Epidemiol 2015; 7: 491-508.

21 Maret-Ouda J, Tao W, Wahlin K, et al. Nordic registry-based cohort studies: possibilities and pitfalls when combining Nordic registry data. Scand J Public Health 2017; 45: 14-19.

22 Schatz M, Cook EF, Joshua A, et al. Risk factors for asthma hospitalizations in a managed care organization: development of a clinical prediction rule. Am J Manag Care 2003; 9: 538-547.

23 Ortqvist AK, Lundholm C, Wettermark B, et al. Validation of asthma and eczema in population-based Swedish drug and patient registers. Pharmacoepidemiol Drug Saf 2013; 22: 850-860.

24 Chung KF, Wenzel SE, Brozek JL, et al. International ERS/ATS guidelines on definition, evaluation and treatment of severe asthma. Eur Respir J 2014; 43: 343-373.

25 Global Initiative for Asthma. Global Strategy for Asthma Management and Prevention, 2018. https:/ginasthma. org/gina-reports/

26 Larsson K, Stallberg B, Lisspers K, et al. Prevalence and management of severe asthma in primary care: an observational cohort study in Sweden (PACEHR). Respir Res 2018; 19: 12.

27 Backman H, Jansson SA, Stridsman C, et al. Severe asthma - a population study perspective. Clin Exp Allergy 2019; 49: 819-828.

28 Hekking PP, Wener RR, Amelink M, et al. The prevalence of severe refractory asthma. J Allergy Clin Immunol 2015; 135: 896-902. 